\title{
AN A POSTERIORI ERROR ESTIMATE FOR MIXED FINITE ELEMENT APPROXIMATIONS OF THE NAVIER-STOKES EQUATIONS
}

\author{
Abdeslam Elakkad, Ahmed Elkhalfi, and Najib Guessous \\ ABSTRACT. In this work, a numerical solution of the incompressible Navi- \\ er-Stokes equations is proposed. The method suggested is based on an \\ algorithm of discretization by mixed finite elements with a posteriori er- \\ ror estimation of the computed solutions. In order to evaluate the per- \\ formance of the method, the numerical results are compared with some \\ previously published works or with others coming from commercial code \\ like Adina system.
}

\section{Introduction}

In modeling flow in porous media, it is essential to use a discretization method which satisfies the physics of the problem, i.e., conserve mass locally and preserve continuity of flux. The Raviart-Thomas Mixed Finite Element (MFE) method of lowest order satisfies these properties. Moreover, both the pressure and the velocity are approximated with the same order of convergence [10], [8]. The discretization of the velocity is based on the properties of RaviartThomas. Other works have been introduced by Brezzi, Fortin, Marini, Dougla and Robert [30], [28], [8]. This method was widely used for the prediction of the behavior of fluid in the hydrocarbons tank.

We were interested in the resolution of the incompressible Navier-Stokes equations in two dimensions on the fields where the numerical problem is well posed with boundary conditions and other aspects of the problem. A discretization by quadrangular finite elements is used. Two iterative methods are used to solve the not-symmetrical discrete system of the Navier-Stokes equations. The biconjugate gradients stabilized method (BICGSTAB) and minimal residual generalized method (GMRES) given in [13], [20]. The technique of preconditioning of the linear systems of big sizes is used to reduce the time of convergence of the iterative methods. This technique of preconditioning has

Received November 25, 2009; Revised August 4, 2010.

2010 Mathematics Subject Classification. 49K05, 49K15, $49 \mathrm{~S} 05$.

Key words and phrases. incompressible Navier-Stokes equations, mixed finite element method, a posteriori error estimates, iterative solvers, Adina system. 
allowed us to accelerate the convergence of the iterative methods independently of the Reynolds number and the number of meshes. Moreover, the methods of Picard or Newton are used to solve the non-linear algebraic systems resulting from the discretization.

In the conforming case, there are several ways to define error estimators by using the residual equation. In particular, for the Stokes problem, M. Ainsworth and J. Oden [2], D. Kay and D. Silvester [23], C. Cartensen and S. A. Fuken [9] and R. Verfurth [31] introduced several error estimators and provided that they are equivalent to the energy norm of the errors. Other works for the stationary Navier-Stokes problem have been introduced in [27], [32], [22], [34], [1], [26], [3].

Section 2 presents the model problem used in this paper. The discretization by mixed finite elements is described in Section 3. Section 4 shows the methods of a posteriori error bounds of the computed solution. Numerical experiments carried out within the framework of this publication and their comparisons with other results are shown in Section 5.

\section{Incompressible Navier-Stokes equations}

We consider the steady-state Navier-Stokes equations for the flow of a Newtonian incompressible viscous fluid with constant viscosity:

$$
\left\{\begin{array}{l}
-\nu \nabla^{2} \vec{u}+\vec{u} \cdot \nabla \vec{u}+\nabla p=\vec{f}, \\
\nabla \cdot \vec{u}=0,
\end{array}\right.
$$

where $\nu>0$ is a given constant called the kinematic viscosity.

$\vec{u}$ is the fluid velocity, $p$ is the pressure field, $\nabla$ is the gradient and $\nabla$. is the divergence operator.

The boundary value problem that is considered is the system (2.1) posed on two or three-dimensional domain $\Omega$, together with boundary conditions on $\partial \Omega=\partial \Omega_{D} \bigcup \partial \Omega_{N}$ given by

$$
\vec{u}=\vec{W} \text { on } \partial \Omega_{D}, \nu \frac{\partial \vec{u}}{\partial n}-\vec{n} p=\overrightarrow{0} \text { on } \partial \Omega_{N},
$$

where $\vec{n}$ denote the outward pointing normal to the boundary.

This system is the basis for computational modeling of the flow of an incompressible Newtonian fluid such as air or water. The presence of the nonlinear convection term $\vec{u} \cdot \nabla \vec{u}$ means that boundary value problems associated with the Navier-Stokes equations can have more than one solution.

We define the spaces:

$$
\begin{gathered}
\hbar^{1}(\Omega)=\left\{u: \Omega \rightarrow \mathbb{R} \mid u, \frac{\partial u}{\partial x}, \frac{\partial u}{\partial y} \in L^{2}(\Omega)\right\}, \\
H_{E}^{1}=\left\{\vec{u} \in \hbar^{1}(\Omega)^{2} \mid \vec{u}=\vec{W} \text { on } \partial \Omega_{D}\right\}, \\
H_{E_{0}}^{1}=\left\{\vec{v} \in \hbar^{1}(\Omega)^{2} \mid \vec{v}=\overrightarrow{0} \text { on } \partial \Omega_{D}\right\},
\end{gathered}
$$




$$
L_{0}^{2}(\Omega)=\left\{q \in L^{2}(\Omega): \int_{\Omega} q d x=0\right\} .
$$

Then the standard weak formulation of the Navier-Stokes flow problem (2.1) and (2.2) is the following:

Find $\vec{u} \in H_{E}^{1}$ and $p \in L_{0}^{2}(\Omega)$ such that

$$
\begin{gathered}
\nu \int_{\Omega} \nabla \vec{u}: \nabla \vec{v}+\int_{\Omega}(\vec{u} \cdot \nabla \vec{u}) \cdot \vec{v}-\int_{\Omega} p(\nabla \cdot \vec{v})=\int_{\Omega} f \cdot \vec{v} \text { for all } \vec{v} \in H_{E_{0}}^{1}, \\
\int_{\Omega} q(\nabla \cdot \vec{u})=0 \text { for all } q \in L_{0}^{2}(\Omega) .
\end{gathered}
$$

Let the bilinear forms $a: H_{E}^{1} \times H_{E}^{1} \longrightarrow \mathbb{R}, b: H_{E}^{1} \times L_{0}^{2}(\Omega) \longrightarrow \mathbb{R}, d: L_{0}^{2} \times L_{0}^{2} \longrightarrow$ $\mathbb{R}$, and the trilinear form $c: H_{E}^{1} \times H_{E}^{1} \times H_{E}^{1} \longrightarrow \mathbb{R}$

$$
\begin{gathered}
a(\vec{u}, \vec{v})=\nu \int_{\Omega} \nabla \vec{u}: \nabla \vec{v}, \\
b(\vec{v}, q)=-\int_{\Omega} q(\nabla \cdot \vec{v}), \\
d(p, q)=\int_{\Omega} p q, \\
c(\vec{z} ; \vec{u}, \vec{v})=\int_{\Omega}(\vec{z} \cdot \nabla \vec{u}) \cdot \vec{v} .
\end{gathered}
$$

Let the subspace of divergence-free velocities be given by

$$
V_{E_{0}}=\left\{\vec{z} \in H_{E_{0}}^{1} ; \nabla \cdot \vec{z}=0 \text { in } \Omega\right\} .
$$

Which induces the norms

$$
\begin{gathered}
\|\vec{v}\|_{a}=a(\vec{u}, \vec{u})^{\frac{1}{2}} \quad \forall \vec{u} \in H_{E_{0}}^{1}, \\
\|q\|_{d}=d(q, q)^{\frac{1}{2}} \quad \forall q \in L_{0}^{2}(\Omega) .
\end{gathered}
$$

Lemma 1. Let $a(.,$.$) and b(.,$.$) be the bilinear forms given by (2.9) and (2.10),$ respectively, and let $c(., .,$.$) the trilinear form given by (2.12). Then$

$$
\begin{gathered}
|a(\vec{v}, \vec{w})| \leq\|\vec{v}\|_{a}\|\vec{w}\|_{a}, \forall \vec{v}, \vec{w} \in H_{E_{0}}^{1}, \\
|b(\vec{v}, q)| \leq \sqrt{2}\|\vec{v}\|_{a}\|q\|_{d}, \forall(\vec{v}, q) \in H_{E_{0}}^{1} \times L_{0}^{2}(\Omega), \\
\sup _{\vec{v} \in H_{E_{0}}^{1}} \frac{b(\vec{v}, q)}{\|\vec{v}\|_{a}} \geq \alpha\|q\|_{d}, \quad \forall q \in L_{0}^{2}(\Omega), \\
c(\vec{z} ; \vec{u}, \vec{v}) \leq \beta|\vec{z}|_{1, \Omega}|\vec{u}|_{1, \Omega}|\vec{v}|_{1, \Omega}, \forall \vec{z}, \vec{v} \in H_{E_{0}}^{1} .
\end{gathered}
$$


The convection term is skew-symmetric: $c(\vec{z} ; \vec{u}, \vec{v})=-c(\vec{z} ; \vec{v}, \vec{u})$ over $V_{E_{0}}$, this mean that

$$
c(\vec{z} ; \vec{u}, \vec{u})=0 \quad \forall \vec{z} \in V_{E_{0}}
$$

Proof. The proof follows from classical results in [18].

We define

$$
\|\vec{f}\|_{*}=\sup _{\vec{v} \in V_{E_{0}}} \frac{(\vec{f}, \vec{v})}{\|\nabla \vec{v}\|} .
$$

Then a well-known (sufficient) condition for uniqueness (see [18, Theorem 2.2]) is that forcing function is small in the sense that

$$
\|\vec{f}\|_{*} \leq \frac{\nu^{2}}{\beta_{*}}
$$

where $\beta_{*}$ is the best possible constant such that (2.19) holds.

Theorem 1. Assume that $\nu$ and $\vec{f} \in L^{2}(\Omega)^{2}$ satisfy the following condition:

$$
\left|(\vec{f}, \vec{v})_{\Omega}\right| \leq \gamma \frac{\nu^{2}}{\beta}|\vec{v}|_{1, \Omega}, \quad \forall \vec{v} \in H_{E_{0}}^{1}
$$

for some fixed number $\gamma \in[0,1)$. Then, there exits an unique solution $(\vec{u}, p) \in$ $H_{E_{0}}^{1} \times L_{0}^{2}(\Omega)$ of (2.7)-(2.8) and it holds

$$
|\vec{u}|_{1, \Omega} \leq \gamma \frac{\nu}{\beta}
$$

Proof. See Theorem 2.4, Chapter IV in [18].

\section{Mixed finite element approximation}

Our goal here is to consider the stationary Navier-Stokes equations with mixed boundary conditions (the Dirichlet boundary condition on a part of the boundary $\partial \Omega_{D}$ and the Neumann one on the remaining part $\partial \Omega_{N}$ ) in a two-dimensional domain and to approximate them by a mixed finite element method.

Mixed finite element discretization of the weak formulation of the NavierStokes equations gives rise to a nonlinear system of algebraic equations. Two classical iterative procedures for solving this system are Newton iteration and Picard iteration.

Let $\tau_{h}, h>0$, be a family of triangulations of $\Omega$. We denote by $h_{T}$ the diameter of a simplex $T$, by $h_{E}$ the diameter of a face $E$ of $T$, and we set $h=\max _{T \in \tau_{h}}\left\{h_{T}\right\}$.

For any $T \in \tau_{h}$ we denote by $\varepsilon(T)$ and $N(T)$ the set of its edges and vertices, respectively.

A discrete weak formulation is defined using finite dimensional spaces $X_{0}^{h} \subset$ $H_{E_{0}}^{1}$ and $M^{h} \subset L_{0}^{2}(\Omega)$. 
Specifically, given a velocity solution spaces $X_{E}^{h} \subset H_{E}^{1}$, the discrete version of (2.7)-(2.8) is: find $\vec{u}_{h} \in X_{E}^{h}$ and $p_{h} \in M^{h}$ such that

$$
\begin{gathered}
\nu \int_{\Omega} \nabla \vec{u}_{h}: \nabla \vec{v}_{h}+\int_{\Omega}\left(\vec{u}_{h} \cdot \nabla \vec{u}_{h}\right) \cdot \vec{v}_{h}-\int_{\Omega} p_{h}\left(\nabla \cdot \vec{v}_{h}\right)=\int_{\Omega} \vec{f} \cdot \vec{v}_{h}, \\
\int_{\Omega} q_{h}\left(\nabla \cdot \vec{u}_{h}\right)=0
\end{gathered}
$$

for all $\vec{v}_{h} \in X_{0}^{h}$ and $q_{h} \in M^{h}$.

We define the appropriate bases for the finite element spaces, leading to a non-linear system of algebraic equations. Linearization of this system using Newton iteration gives the finite dimensional system: find $\delta \vec{u}_{h} \in X_{0}^{h}$ and $\delta p_{h} \in M^{h}$ such that

$$
\begin{gathered}
c\left(\delta \vec{u}_{h} ; \vec{u}_{h}, \vec{v}_{h}\right)+c\left(\vec{u}_{h} ; \delta \vec{u}_{h}, \vec{v}_{h}\right)+\nu \int_{\Omega} \nabla \delta \vec{u}_{h}: \\
\nabla \vec{v}_{h}-\int_{\Omega} \delta p_{h}\left(\nabla \cdot \vec{v}_{h}\right)=R_{k}\left(\vec{v}_{h}\right), \\
\int_{\Omega} q_{h}\left(\nabla \cdot \delta \vec{u}_{h}\right)=r_{k}\left(q_{h}\right)
\end{gathered}
$$

for all $\vec{v}_{h} \in X_{0}^{h}$ and $q_{h} \in M^{h}$. Here, $R_{k}\left(\vec{v}_{h}\right)$ and $r_{k}\left(q_{h}\right)$ are the non-linear residuals associated with the discrete formulations (3.1) and (3.2).

To define the corresponding linear algebra problem, we use a set of vectorvalued basis functions $\left\{\vec{\varphi}_{j}\right\}$, so that

$$
\vec{u}_{h}=\sum_{j=1}^{n_{u}} u_{j} \vec{\varphi}_{j}+\sum_{j=n_{u}+1}^{n_{u}+n_{\partial}} u_{j} \vec{\varphi}_{j}, \delta \vec{u}_{h}=\sum_{j=1}^{n_{u}} \Delta u_{j} \vec{\varphi}_{j}
$$

and we fix the coefficients $u_{j}: j=n_{u}+1, \ldots, n_{u}+n_{\partial}$, so that the second term interpolates the boundary data on $\partial \Omega_{D}$.

We introduce a set of pressure basis functions $\left\{\Psi_{k}\right\}$ and set

$$
p_{h}=\sum_{k=1}^{n_{p}} p_{k} \Psi_{k}, \delta p_{h}=\sum_{k=1}^{n_{p}} \Delta p_{k} \Psi_{k},
$$

where $n_{u}$ and $n_{p}$ are the numbers of velocity and pressure basis functions, respectively.

We obtain a system of linear equations

$$
\left(\begin{array}{cc}
\nu A+N+W & { }^{t} B \\
B & 0
\end{array}\right)\left(\begin{array}{c}
\Delta U \\
\Delta P
\end{array}\right)=\left(\begin{array}{l}
f \\
g
\end{array}\right)
$$

This system is referred to as the discrete Newton problem.

The matrix $A$ is the vector Laplacian matrix and $B$ is the divergence matrix

$$
A=\left[a_{i j}\right], a_{i j}=\int_{\Omega} \nabla \vec{\varphi}_{i}: \nabla \vec{\varphi}_{j}
$$




$$
B=\left[b_{k j}\right], b_{k j}=-\int_{\Omega} \Psi_{k} \nabla \cdot \vec{\varphi}_{j}
$$

for $i$ and $j=1, \ldots, n_{u}$ and $k=1, \ldots, n_{p}$.

The vector-convection matrix $N$ and the Newton derivative matrix $W$ are given by

$$
\begin{gathered}
N=\left[n_{i j}\right], n_{i j}=\int_{\Omega}\left(\vec{u}_{h} \cdot \nabla \vec{\varphi}_{j}\right) \cdot \vec{\varphi}_{i}, \\
W=\left[W_{i j}\right], W_{i j}=\int_{\Omega}\left(\vec{\varphi}_{j} \cdot \nabla \vec{u}_{h}\right) \cdot \vec{\varphi}_{i}
\end{gathered}
$$

for $i$ and $j=1, \ldots, n_{u}$. The Newton derivative matrix is symmetric.

The right-hand side vectors in (3.6) are the non-linear residuals associated with the current discrete solution $\vec{u}_{h}$ and $p_{h}$.

$f=\left[f_{i}\right], f_{i}=\int_{\Omega} f \cdot \vec{\varphi}_{i}-\int_{\Omega}\left(\vec{u}_{h} \cdot \nabla \vec{u}_{h}\right) \cdot \vec{\varphi}_{i}-\nu \int_{\Omega} \nabla \vec{u}_{h}: \nabla \vec{\varphi}_{i}+\int_{\Omega} p_{h}\left(\nabla \cdot \vec{\varphi}_{i}\right)$,

$$
g=\left[g_{k}\right], g_{k}=-\int_{\Omega} \Psi_{k}\left(\nabla \cdot \vec{u}_{h}\right) .
$$

For Picard iteration, we give the discrete problem

$$
\left(\begin{array}{cc}
\nu A+N & { }^{t} B \\
B & 0
\end{array}\right)\left(\begin{array}{c}
\Delta U \\
\Delta P
\end{array}\right)=\left(\begin{array}{c}
f \\
g
\end{array}\right) .
$$

The lowest order mixed approximations like $Q_{1}-P_{0}$ and $Q_{1}-Q_{1}$ are unstable. We use a stabilized element pair $Q_{1}-P_{0}$, this is the most famous example of an unstable element pair, using bilinear approximation for velocity and a constant approximation for the pressure.

We use two iterative methods for solving the nonsymmetric systems: The generalized minimum residual method (GMRES) and BiConjugate Gradients Stabilized Method (BICGSTAB) [13], [20].

Preconditioning is a technique used to enhance the convergence of an iterative method to solve a large linear systems iteratively. Instead of solving a system $\Lambda x=b$, one solves a system $P^{-1} \Lambda x=P^{-1} b$, where $P$ is the preconditioned. A good preconditioned should lead to fast convergence of the Krylov method. Furthermore, systems of the form $P z=r$ should be easy to solve.

For the Navier-Stokes equations, the objective is to design a preconditioned that increases the convergence of an iterative method independent of the Reynolds number and number of grid points. We use a least-squares commutator preconditioning [29], [16].

\section{A posteriori error bounds}

In this section we consider a posteriori error estimators for the stationary Navier-Stokes equation. The estimator requires the solution of local Poisson problems, and we derive a global upper bound for the error. 
Throughout this section we restrict attention to the simplest case of enclosed flow with $\partial \Omega=\partial \Omega_{D}$ and $\vec{W}=\overrightarrow{0}$.

Given the continuous functional $l: H_{E}^{1} \longrightarrow \mathbb{R}, l(\vec{v})=\int_{\Omega} \vec{f} \cdot \vec{v}$; the underlying weak formulation (2.7)-(2.8) may be restated as: Find $\vec{u} \in H_{E_{0}}^{1}$ and $p \in L_{0}^{2}(\Omega)$ such that

$$
\left\{\begin{array}{l}
a(\vec{u}, \vec{v})+c(\vec{u} ; \vec{u}, \vec{v})+b(\vec{v}, p)=l(\vec{v}) \text { for all } \vec{v} \in H_{E_{0}}^{1}, \\
b(\vec{u}, q)=0 \text { for all } q \in L_{0}^{2}(\Omega) .
\end{array}\right.
$$

With a conforming mixed approximation, the corresponding discrete problem (3.1)-(3.2) is given by: Find $\vec{u}_{h} \in X_{0}^{h}$ and $p_{h} \in M^{h}$ such that

$$
\left\{\begin{array}{l}
a\left(\vec{u}_{h}, \vec{v}_{h}\right)+c\left(\vec{u}_{h} ; \vec{u}_{h}, \vec{v}_{h}\right)+b\left(\vec{v}_{h}, p_{h}\right)=l\left(\vec{v}_{h}\right) \text { for all } \vec{v}_{h} \in X_{0}^{h} \\
b\left(\vec{u}_{h}, q_{h}\right)=0 \text { for all } q_{h} \in M^{h}
\end{array}\right.
$$

Our aim is to bound $\left\|\vec{u}-\vec{u}_{h}\right\|_{X}$ and $\left\|p-p_{h}\right\|_{M}$ with respect to the energy norm for the velocity $\|\vec{v}\|_{X}=\|\nabla \vec{v}\|$ and the quotient norm for the pressure $\|p\|_{M}=\|p\|_{0, \Omega}$.

Let the symmetric bilinear form

$$
B((\vec{u}, p) ;(\vec{v}, q))=a(\vec{u}, \vec{v})+b(\vec{u}, q)+b(\vec{v}, p),
$$

and introducing the functional $F((\vec{v}, q))=l(\vec{v})$ associated with the forcing term, we have that the errors $\vec{e}=\vec{u}-\overrightarrow{u_{h}} \in H_{E_{0}}^{1}$ and $\varepsilon=p-p_{h} \in L_{0}^{2}(\Omega)$ associated with (4.1) and (4.2) satisfy

$$
\begin{aligned}
B((\vec{e}, \varepsilon) ;(\vec{v}, q))= & B\left(\left(\vec{u}-\vec{u}_{h}, p-p_{h}\right) ;(\vec{v}, q)\right) \\
= & B((\vec{u}, p) ;(\vec{v}, q))-B\left(\left(\vec{u}_{h}, p_{h}\right) ;(\vec{v}, q)\right) \\
= & F((\vec{v}, q))-c(\vec{u} ; \vec{u}, \vec{v})-B\left(\left(\vec{u}_{h}, p_{h}\right) ;(\vec{v}, q)\right) \\
= & -c(\vec{u} ; \vec{u}, \vec{v})+l(\vec{v})-a\left(\vec{u}_{h}, \vec{v}\right)-b\left(\vec{v}, p_{h}\right) \\
& -b\left(\vec{u}_{h}, q\right)
\end{aligned}
$$

for all $(\vec{v}, q) \in H_{E_{0}}^{1} \times L_{0}^{2}(\Omega)$.

We note that

$$
\begin{aligned}
D\left(\vec{u}_{h}, \vec{e}, \vec{v}\right) & =c\left(\vec{e}+\vec{u}_{h} ; \vec{e}+\vec{u}_{h}, \vec{v}\right)-c\left(\vec{u}_{h} ; \vec{u}_{h}, \vec{v}\right) \\
& =c(\vec{u} ; \vec{u}, \vec{v})-c\left(\vec{u}_{h} ; \vec{u}_{h}, \vec{v}\right) .
\end{aligned}
$$

We combine this with (4.4), we get

$$
\begin{aligned}
& B((\vec{e}, \varepsilon) ;(\vec{v}, q))+D\left(\vec{u}_{h}, \vec{e}, \vec{v}\right) \\
= & -c\left(\vec{u}_{h} ; \vec{u}_{h}, \vec{v}\right)+l(\vec{v})-a\left(\vec{u}_{h}, \vec{v}\right)-b\left(\vec{v}, p_{h}\right)-b\left(\vec{u}_{h}, q\right) .
\end{aligned}
$$

We define the stress jump across edge or face $E$ adjoining elements $T$ and $S$ :

$$
\left[\left[\nu \nabla \vec{u}_{h}-p_{h} \vec{I}\right]\right]=\left(\left.\left(\nu \nabla \vec{u}_{h}-p_{h} \vec{I}\right)\right|_{T}-\left.\left(\nu \nabla \vec{u}_{h}-p_{h} \vec{I}\right)\right|_{S}\right) \vec{n}_{E, T},
$$

where $\vec{n}_{E, T}$ is the outward pointing normal. 
We define the equidistributed stress jump operator

$$
\vec{R}_{E}^{*}=\frac{1}{2}\left[\left[\nu \nabla \vec{u}_{h}-p_{h} \vec{I}\right]\right]
$$

and the interior residuals

$$
\vec{R}_{T}=\left.\left\{\vec{f}+\nu \nabla^{2} \vec{u}_{h}-\vec{u}_{h} \cdot \nabla \vec{u}_{h}-\nabla p_{h}\right\}\right|_{T}
$$

and $R_{T}=\left.\left\{\nabla \cdot \vec{u}_{h}\right\}\right|_{T}$, we find that the errors $\vec{e} \in H_{E_{0}}^{1}$ and $\varepsilon \in L_{0}^{2}(\Omega)$ satisfy the non-linear equation

$$
\begin{aligned}
& B((\vec{e}, \varepsilon) ;(\vec{v}, q))+D\left(\vec{u}_{h}, \vec{e}, \vec{v}\right) \\
= & \sum_{T \in \tau_{h}}\left[\left(\vec{R}_{T}, \vec{v}\right)_{T}-\sum_{E \in \varepsilon(T)}\left\langle\vec{R}_{E}^{*}, \vec{v}\right\rangle_{E}+\left(R_{T}, q\right)_{T}\right]
\end{aligned}
$$

for all $(\vec{v}, q) \in H_{E_{0}}^{1} \times L_{0}^{2}(\Omega)$.

The error characterization (4.7) is the starting point for a posteriori error analysis. The crucial question here is to determine the best way of handling the non-linear term on the left-hand side of (4.7). We note that

$$
D\left(\vec{u}_{h}, \vec{e}, \vec{v}\right)=c(\vec{e} ; \vec{e}, \vec{v})+c\left(\vec{e} ; \vec{u}_{h}, \vec{v}\right)+c\left(\vec{u}_{h} ; \vec{e}, \vec{v}\right) .
$$

The problem (4.7) can be approximated by the linear problem: find $\vec{e} \in H_{E_{0}}^{1}$ and $\varepsilon \in L_{0}^{2}(\Omega)$ such that

$$
\begin{aligned}
& c\left(\vec{e} ; \vec{u}_{h}, \vec{v}\right)+c\left(\vec{u}_{h} ; \vec{e}, \vec{v}\right)+\nu \int_{\Omega} \nabla \vec{e} \cdot \nabla \vec{v}-\int_{\Omega} \varepsilon(\nabla \cdot \vec{v}) \\
= & \sum_{T \in \tau_{h}}\left[\left(\vec{R}_{T}, \vec{v}\right)_{T}-\sum_{E \in \varepsilon(T)}\left\langle\vec{R}_{E}^{*}, \vec{v}\right\rangle_{E}\right]-\int_{\Omega} q(\nabla \cdot \vec{e}) \\
= & \sum_{T \in \tau_{h}}\left(R_{T}, q\right)_{T} .
\end{aligned}
$$

This system corresponds to a Newton linearization about the discrete velocity solution $\vec{u}_{h}$. We shall concentrate on the stabilized $Q_{1}-P_{0}$ or $P_{1}-P_{0}$ approximation methods in two dimensions. Notice that for either of these low order approximations, the divergence residual $R_{T}$ is piecewise constant and the stress jump term $\vec{R}_{E}^{*}$ is piecewise linear. The other element residual is given by $\vec{R}_{T}=\left.\left\{\vec{f}-\vec{u}_{h} \cdot \nabla \vec{u}_{h}\right\}\right|_{T}$.

For each $T \in \tau_{h}$ let

$$
V_{T}=\left\{\vec{v} \in H^{1}(T) \times H^{1}(T): \vec{v}=\overrightarrow{0} \text { on } \partial \Omega \cap \partial T\right\} .
$$

We find $\vec{e}_{T} \in V_{T}$ satisfying the uncoupled poisson problems

$$
\nu\left(\nabla \vec{e}_{T}, \nabla \vec{v}\right)_{T}=\left(\vec{R}_{T}, \vec{v}\right)_{T}-\sum_{E \in \varepsilon(T)}\left\langle\vec{R}_{E}^{*}, \vec{v}\right\rangle_{E} \text { for all } \vec{v} \in V_{T} .
$$

With $\varepsilon_{T}=\nabla \cdot \vec{u}_{h}$, the local error estimator is given by the combination of the energy norm of the velocity error and the $L^{2}$ norm of the element divergence 
error, that is

$$
\eta_{T}^{2}=\left\|\nabla \vec{e}_{T}\right\|_{T}^{2}+\left\|\varepsilon_{T}\right\|_{T}^{2}=\left\|\nabla \vec{e}_{T}\right\|_{T}^{2}+\left\|\nabla \cdot \vec{u}_{h}\right\|_{T}^{2},
$$

and the global error estimator is

$$
\eta=\left(\sum_{T \in \tau_{h}} \eta_{T}^{2}\right)^{\frac{1}{2}}
$$

We define a pair $(\vec{\Phi}, \psi) \in H_{E_{0}}^{1} \times L_{0}^{2}(\Omega)$ to be the Ritz projection of the modified residuals

(4.11) $\quad a(\vec{\Phi}, \vec{v})+d(\psi, q)=a(\vec{e}, \vec{v})+b(\vec{e}, q)+b(\vec{v}, \varepsilon)+D\left(\vec{u}_{h} ; \vec{e}, \vec{v}\right)$

for all $(\vec{v}, q) \in H_{E_{0}}^{1} \times L_{0}^{2}(\Omega)$, where $\vec{e}=\vec{u}-\vec{u}_{h}, \varepsilon=p-p_{h}$ and $D\left(\vec{u}_{h} ; \vec{e}, \vec{v}\right)$ $=c(\vec{u} ; \vec{u}, \vec{v})-c\left(\vec{u}_{h} ; \vec{u}_{h}, \vec{v}\right)$.

The problem (4.11) is equivalent to find $(\vec{\Phi}, \psi) \in H_{E_{0}}^{1} \times L_{0}^{2}(\Omega)$ satisfying (4.12)

$a(\vec{\Phi}, \vec{v})+d(\psi, q)=(\vec{f}, \vec{v})_{\Omega}-a\left(\vec{u}_{h}, \vec{v}\right)-b\left(\vec{u}_{h}, q\right)-b\left(\vec{v}, p_{h}\right)-c\left(\vec{u}_{h} ; \vec{u}_{h}, \vec{v}\right)$ for all $(\vec{v}, q) \in H_{E_{0}}^{1} \times L_{0}^{2}(\Omega)$.

Next, we establish an equivalence between the norms of $(\vec{e}, \varepsilon) \in H_{E_{0}}^{1} \times L_{0}^{2}(\Omega)$ and the norms of the solution $(\vec{\Phi}, \psi) \in H_{E_{0}}^{1} \times L_{0}^{2}(\Omega)$ of (4.11).

Theorem 2. Let the conditions of Theorem 1 hold. There exist positive constants $K_{1}$ and $K_{2}$, independent of $h$, such that

$$
K_{1}\left\{\|\vec{\Phi}\|_{a}^{2}+\|\psi\|_{d}^{2}\right\} \leq\|\vec{e}\|_{a}^{2}+\|\varepsilon\|_{d}^{2} \leq K_{2}\left\{\|\vec{\Phi}\|_{a}^{2}+\|\psi\|_{d}^{2}\right\} .
$$

Proof. First, note that using (2.19) and (2.24), we have that for $\vec{v} \in H_{E_{0}}^{1}$

$$
\begin{aligned}
D\left(\vec{u}_{h} ; \vec{e}, \vec{v}\right) & =c(\vec{u} ; \vec{u}, \vec{v})-c\left(\vec{u}_{h} ; \vec{u}_{h}, \vec{v}\right) \\
& =c(\vec{u} ; \vec{e}, \vec{v})+c(\vec{e} ; \vec{u} h, \vec{v}) \\
& \leq \beta\left\{|\vec{u}|_{1, \Omega}|\vec{e}|_{1, \Omega}|\vec{v}|_{1, \Omega}+|\vec{e}|_{1, \Omega}\left|\vec{u}_{h}\right|_{1, \Omega}|\vec{v}|_{1, \Omega}\right\} \\
& \leq \beta\left\{2|\vec{u}|_{1, \Omega}|\vec{e}|_{1, \Omega}|\vec{v}|_{1, \Omega}+|\vec{e}|_{1, \Omega}^{2}|\vec{v}|_{1, \Omega}\right\} \\
& \leq \beta\left\{2 \gamma \frac{\nu}{\beta}|\vec{e}|_{1, \Omega}|\vec{v}|_{1, \Omega}+|\vec{e}|_{1, \Omega}^{2}|\vec{v}|_{1, \Omega}\right\} \\
& \leq\left\{2 \gamma+\frac{\beta}{\nu}|\vec{e}|_{1, \Omega}\right\}\|\vec{e}\|_{a}\|\vec{v}\|_{a} .
\end{aligned}
$$

Furthermore, using (2.20) and following above steps closely it holds

$$
D\left(\vec{u}_{h} ; \vec{e}, \vec{e}\right) \leq\left\{\gamma+\frac{\beta}{\nu}|\vec{e}|_{1, \Omega}\right\}\|\vec{e}\|_{a}^{2} .
$$

Next, the upper and lower bound are addressed separately.

Upper bound: From the equation (2.18) and setting $q=0$ in (4.11), (2.16) and (4.13), there holds

$$
\alpha\|\varepsilon\|_{d} \leq \sup _{\vec{v} \in H_{E_{0}}^{1}} \frac{b(\vec{v}, \varepsilon)}{\|\vec{v}\|_{a}}
$$




$$
\begin{aligned}
& =\sup _{\vec{v} \in H_{E_{0}}^{1}} \frac{\left|a(\vec{\Phi}, \vec{v})-a(\vec{e}, \vec{v})-D\left(\vec{u}_{h} ; \vec{e}, \vec{v}\right)\right|}{\|\vec{v}\|_{a}} \\
& \leq\|\vec{\Phi}\|_{a}+\left\{3+\frac{\beta}{\nu}|\vec{e}|_{1, \Omega}\right\}\|\vec{e}\|_{a} .
\end{aligned}
$$

In addition, by picking $q=0$ and $\vec{v}=\vec{e}$ in (4.11) and using (4.14) we arrive at

$$
\begin{aligned}
\|\vec{e}\|_{a}^{2} & =a(\vec{\Phi}, \vec{e})-b(\vec{e}, \varepsilon)-D\left(\vec{u}_{h} ; \vec{e}, \vec{e}\right) \\
& =a(\vec{\Phi}, \vec{e})-d(\psi, \varepsilon)-D\left(\vec{u}_{h} ; \vec{e}, \vec{e}\right) \\
& \leq\|\vec{\Phi}\|_{a}\|\vec{e}\|_{a}+\|\psi\|_{d}\|\varepsilon\|_{d}+\left\{\gamma+\frac{\beta}{\nu}|\vec{e}|_{1, \Omega}\right\}\|\vec{e}\|_{a}^{2},
\end{aligned}
$$

and we gather (4.15) and (4.16) to obtain

$$
\begin{aligned}
\|\vec{e}\|_{a}^{2} \leq & \frac{1}{\alpha}\|\vec{\Phi}\|_{a}\|\psi\|_{d}+\|\vec{e}\|_{a}\left\{\|\vec{\Phi}\|_{a}+\frac{1}{\alpha}\left(3+\frac{\beta}{\nu}|\vec{e}|_{1, \Omega}\right)\|\psi\|_{d}\right\}+ \\
& \|\vec{e}\|_{a}^{2}\left\{\gamma+\frac{\beta}{\nu}|\vec{e}|_{1, \Omega}\right\} .
\end{aligned}
$$

We assume $|\vec{e}|_{1, \Omega}$ to be sufficiently small in a way that there exists $\epsilon>0$ such that (see [27] for a related assumption)

$$
\gamma+\frac{\epsilon^{2}}{2}+\frac{\beta}{\nu}|\vec{e}|_{1, \Omega}=\delta<1
$$

The existence of such of $\epsilon$ along with the inequality $x y \leq \frac{\epsilon^{2} x^{2}}{2}+\frac{y^{2}}{2 \epsilon^{2}}$ for all $x, y>0$, imply

$$
\begin{aligned}
\|\vec{e}\|_{a}^{2} \leq & \frac{1}{\alpha}\|\psi\|_{d}\|\| \vec{\Phi}\left\|_{a}+\frac{\epsilon^{2}}{2}\right\| \vec{e} \|_{a}^{2}+\frac{1}{2 \epsilon^{2}}\left\{\|\vec{\Phi}\|_{a}+\frac{1}{\alpha}\left(3+\frac{\beta}{\nu}|\vec{e}|_{1, \Omega}\right)\|\psi\|_{d}\right\}^{2} \\
& +\left\{\gamma+\frac{\beta}{\nu}|\vec{e}|_{1, \Omega}\right\}\|\vec{e}\|_{a}^{2} \\
= & \frac{1}{\alpha}\|\psi\|_{d}\|\vec{\Phi}\|_{a}+\delta\|\vec{e}\|_{a}^{2}+\frac{1}{2 \epsilon^{2}}\left\{\|\vec{\Phi}\|_{a}+\frac{1}{\alpha}\left(3+\frac{\beta}{\nu}|\vec{e}|_{1, \Omega}\right)\|\psi\|_{d}\right\}^{2} \\
\leq & \frac{1}{2 \alpha}\left[\|\psi\|_{d}^{2}+\|\vec{\Phi}\|_{a}^{2}\right]+\delta\|\vec{e}\|_{a}^{2}+\frac{1}{\epsilon^{2}}\left\{\|\vec{\Phi}\|_{a}^{2}+\frac{1}{\alpha^{2}}\left(3+\frac{\beta}{\nu}|\vec{e}|_{1, \Omega}\right)^{2}\|\psi\|_{d}^{2}\right\} \\
(4.19) \leq & {\left[\frac{1}{2 \alpha}+\frac{1}{\varepsilon^{2}}\right]\|\vec{\Phi}\|_{a}^{2}+\delta\|\vec{e}\|_{a}^{2}+\frac{1}{\alpha}\left\{\frac{1}{2}+\frac{1}{\epsilon^{2} \alpha}\left(3+\frac{\beta}{\nu}|\vec{e}|_{1, \Omega}\right)^{2}\right\}\|\psi\|_{d}^{2} . }
\end{aligned}
$$

Now, from the previous inequality and (4.18) we get

$$
\|\vec{e}\|_{a}^{2} \leq \frac{1}{1-\delta}\left[\frac{1}{2 \alpha}+\frac{1}{\epsilon^{2}}\right]\|\vec{\Phi}\|_{a}^{2}+\frac{1}{1-\delta} \frac{1}{\alpha}\left\{\frac{1}{2}+\frac{1}{\epsilon^{2} \alpha}\left(3+\frac{\beta}{\nu}|\vec{e}|_{1, \Omega}\right)^{2}\right\}\|\psi\|_{a}^{2} .
$$

Finally, the upper bound follows from the above inequality and (4.15).

Lower bound: Taking $\vec{v}=\vec{\Phi}$, and $q=0$ in (4.11), and using (2.17) and (4.13), it holds

$$
\begin{aligned}
\|\vec{\Phi}\|_{a}^{2} & =a(\vec{\Phi}, \vec{\Phi}) \\
& =a(\vec{e}, \vec{\Phi})+b(\vec{\Phi}, \varepsilon)+D\left(\vec{u}_{h} ; \vec{e}, \vec{\Phi}\right)
\end{aligned}
$$




$$
\begin{aligned}
& \leq\|\vec{e}\|_{a}\|\vec{\Phi}\|_{a}+\sqrt{2}\|\vec{\Phi}\|_{a}\|\varepsilon\|_{d}+\left\{2 \gamma+\frac{\beta}{\nu}|\vec{e}|_{1, \Omega}\right\}\|\vec{e}\|_{a}\|\vec{\Phi}\|_{a} \\
& \leq \sqrt{2}\|\vec{\Phi}\|_{a}\|\varepsilon\|_{d}+\left\{3+\frac{\beta}{\nu}|\vec{e}|_{1, \Omega}\right\}\|\vec{e}\|_{a}\|\vec{\Phi}\|_{a},
\end{aligned}
$$

then,

$$
\|\vec{\Phi}\|_{a} \leq\left\{3+\frac{\beta}{\nu}|\vec{e}|_{1, \Omega}\right\}\|\vec{e}\|_{a}+\sqrt{2}\|\varepsilon\|_{d} .
$$

Next, setting $\vec{v}=0$ and $q=\psi$ in (4.11), and using (2.17), we end up with

$$
\|\psi\|_{d}^{2}=d(\psi, \psi)=b(\vec{e}, \psi) \leq \sqrt{2}\|\vec{e}\|_{a}\|\psi\|_{a} \leq\|\vec{e}\|_{a}^{2}+\frac{1}{2}\|\psi\|_{d}^{2},
$$

which implies

$$
\|\psi\|_{d}^{2} \leq 2\|\vec{e}\|_{a}^{2}
$$

Hence, we gather (4.20) and (4.21) to get

$$
\|\vec{\Phi}\|_{a}^{2}+\|\psi\|_{d}^{2} \leq 2\left[1+\left\{3+\frac{\beta}{\nu}|\vec{e}|_{1, \Omega}\right\}^{2}\right]\|\vec{e}\|_{a}^{2}+4\|\varepsilon\|_{d}^{2}
$$

Finally, from the bound (4.18) for $\epsilon$, the following inequality holds

$$
3+\frac{\epsilon^{2}}{2}+\frac{\beta}{\nu}|\vec{e}|_{1, \Omega} \leq 4,
$$

we obtain

$$
\|\vec{\Phi}\|_{a}^{2}+\|\psi\|_{d}^{2} \leq 34\left\{\|\vec{e}\|_{a}^{2}+\|\varepsilon\|_{d}^{2}\right\}
$$

and the result follows.

Remark. The auxiliar problem (4.11) is decoupled. In fact, if one set $\vec{v}=\overrightarrow{0}$ in (4.12), then the problem (4.11) becomes

$$
d(\psi, q)=-b\left(\vec{u}_{h}, q\right),
$$

which leads to

$$
\psi=\nabla \cdot \vec{u}_{h} .
$$

Hence, if we pick in the sequel the test functions $(\vec{v}, 0)$ in $(4.12)$ we arrive at

$$
a(\vec{\Phi}, \vec{v})=l(\vec{v})-a\left(\vec{u}_{h}, \vec{v}\right)-b\left(\vec{v}, p_{h}\right)-c\left(\vec{u}_{h} ; \vec{u}_{h}, \vec{v}\right) .
$$

Using integration by parts in (4.26) we arrive at

$$
\nu(\nabla \vec{\Phi}, \nabla \vec{v})_{\Omega}=\sum_{T \in \tau_{h}}\left[\left(\vec{R}_{T}, \vec{v}\right)_{T}-\sum_{E \in \varepsilon(T)}\left\langle\vec{R}_{E}^{*}, \vec{v}\right\rangle_{E}\right]
$$

for all $(\vec{v}, q) \in H_{E_{0}}^{1} \times L_{0}^{2}(\Omega)$, where $\vec{R}_{E}^{*}$ and $\vec{R}_{T}$ defined in (4.5) and (4.6) respectively. 
The local velocity space on each $T \in \tau_{h}$ is

$$
V_{T}=\left\{\vec{v} \in H^{1}(T) \times H^{1}(T): \vec{v}=\overrightarrow{0} \text { on } \partial \Omega \cap \partial T\right\},
$$

and the local pressure space is

$$
Q_{T}=L^{2}(T) .
$$

Let the bilinear form $b_{T}: V_{T} \times Q_{T} \longrightarrow \mathbb{R}$ and the trilinear form $c_{T}: V_{T} \times V_{T} \times$ $V_{T} \longrightarrow \mathbb{R}:$

$$
\begin{aligned}
& b(\vec{v}, q)=\sum_{T \in \tau_{h}} b_{T}(\vec{v}, q), \quad b_{T}(\vec{v}, q)=-\int_{T} q(\nabla \cdot \vec{v}), \forall q \in Q_{T}, \forall \vec{v} \in V_{T}, \\
& \quad c_{T}(\vec{z} ; \vec{u}, \vec{v})=\int_{T}(\vec{z} \cdot \nabla \vec{u}) \cdot \vec{v}, \quad \forall(\vec{z}, \vec{u}, \vec{v}) \in V_{T} \times V_{T} \times V_{T} .
\end{aligned}
$$

Similarly, $l_{T}: V_{T} \longrightarrow \mathbb{R}$ is defined by

$$
l_{T}(\vec{v})=\int_{T} \vec{f} \vec{v}, \forall \vec{v} \in V_{T} .
$$

We introduce the stresslike tensor $\sigma(v, q)$ formally defined to be

$$
\sigma_{i j}(v, q)=\nu \frac{\partial v_{i}}{\partial x_{j}}-q \delta_{i j},
$$

where $\delta_{i j}$ is the Kronecker symbol.

Let $\vec{\Phi}_{T} \in V_{T}$ be such that

$$
\begin{aligned}
a\left(\vec{\Phi}_{T}, \vec{v}\right)= & l_{T}(\vec{v})-a_{T}\left(\vec{u}_{h}, \vec{v}\right)-b_{T}\left(\vec{v}, p_{h}\right)-c_{T}\left(\vec{u}_{h} ; \vec{u}_{h}, \vec{v}\right) \\
& +\oint_{\partial T}\left\langle\vec{n}_{T} \cdot \sigma\left(\vec{u}_{h}, p_{h}\right)\right\rangle \cdot \vec{v} d s
\end{aligned}
$$

for all $\vec{v}_{T} \in V_{T}$, where the boundary data are chosen to respect the equilibration condition,

$0=l_{T}(r)-a_{T}\left(\vec{u}_{h}, r\right)-b_{T}\left(r, p_{h}\right)-c_{T}\left(\vec{u}_{h} ; \vec{u}_{h}, r\right)+\oint_{\partial T}\left\langle\vec{n}_{T} \cdot \sigma\left(\vec{u}_{h}, p_{h}\right)\right\rangle \cdot r d s$, where $r$ is any constant flow field.

Then the error of the finite element approximation of the Navier-Stokes equation (4.1) satisfies the following bound (See Theorem 5 in [27])

$$
\|\vec{\Phi}\|_{a}^{2}+\|\psi\|_{d}^{2} \leq \sum_{T \in \tau_{h}} \eta_{T}^{2}
$$

where

$$
\eta_{T}=\left\{\left\|\nabla \vec{\Phi}_{T}\right\|_{T}^{2}+\left\|\nabla \cdot \vec{u}_{h}\right\|_{T}^{2}\right\}^{\frac{1}{2}}
$$

Theorem 2 and (4.34) now yield the following a posteriori error estimate: 
Theorem 3. Let the conditions of Theorem 1 hold. There exists a constant $C>0$ such that

$$
\left\|\nabla\left(\vec{u}-\vec{u}_{h}\right)\right\|^{2}+\left\|p-p_{h}\right\|_{0, \Omega}^{2} \leq C\left(\sum_{T \in \tau_{h}} \eta_{T}^{2}\right) .
$$

\section{Numerical simulation}

In this section, some numerical results of calculations with mixed finite element method and ADINA system will be presented. Using our solver, we run two traditional test problems (driven cavity flow [14], [15], [6], [24], [20], [17], [25], [21] and Backward-facing step problem [29], [19]) with a number of different model parameters by increasing Reynolds number, and we discuss the performance of our code.

The computational results have been computed using the IFISS software from http://www.manchester.ac.uk/ifiss.

Example 1. Square domain, regularized cavity boundary condition.

This is a classic test problem used in fluid dynamics, known as driven-cavity flow. It is a model of the flow in a square cavity with the lid moving from left to right. Let the computational model: $\left\{y=1 ;-1 \leq x \leq 1 / u_{x}=1-x^{4}\right\}$, a regularized cavity.

The streamlines are computed from the velocity solution by solving the poisson equation numerically subject to a zero Dirichlet boundary condition.

We have solved the problem for three different Reynold's numbers: 100, 600, and 2000. Fig. 2. Uniform streamline plot with MFE (left), and uniform streamline plot computed with ADINA system (right) using $Q_{1}-P_{0}$ approximation, $32 \times 32$ square grid and Reynolds number $R e=600$.

The solution shown in Figure 3 corresponds to a Reynolds number of 2000. The particles in the body of the fluid move in a circular trajectory. Steady flow in a two dimensional cavity is not stable for Reynolds number much greater than $10^{4}$. Indeed, we have made calculations for Reynolds number $10^{4}$, in addition, our code does not converge because the turbulence phenomena is not taken into account in our model. At a critical Reynolds number (approximately $13,000)$ the flow pattern develops into a time-periodic state with "waves" running around the cavity walls.

TABLE 1. Estimated errors for regularized driven cavity flow using $Q_{1}-P_{0}$ approximation for the flow with Reynolds number $R e=100$.

\begin{tabular}{c|c|c|} 
& $\left\|\nabla \cdot \vec{u}_{h}\right\|_{\Omega}$ & $\eta$ \\
\hline $8 \times 8$ & $8.704739 \mathrm{e}-002$ & $1.720480 \mathrm{e}+000$ \\
$16 \times 16$ & $3.115002 \mathrm{e}-002$ & $1.084737 \mathrm{e}+000$ \\
$32 \times 32$ & $9.545524 \mathrm{e}-003$ & $5.919904 \mathrm{e}-001$ \\
$64 \times 64$ & $2.676623 \mathrm{e}-003$ & $3.160964 \mathrm{e}-001$ \\
\hline
\end{tabular}



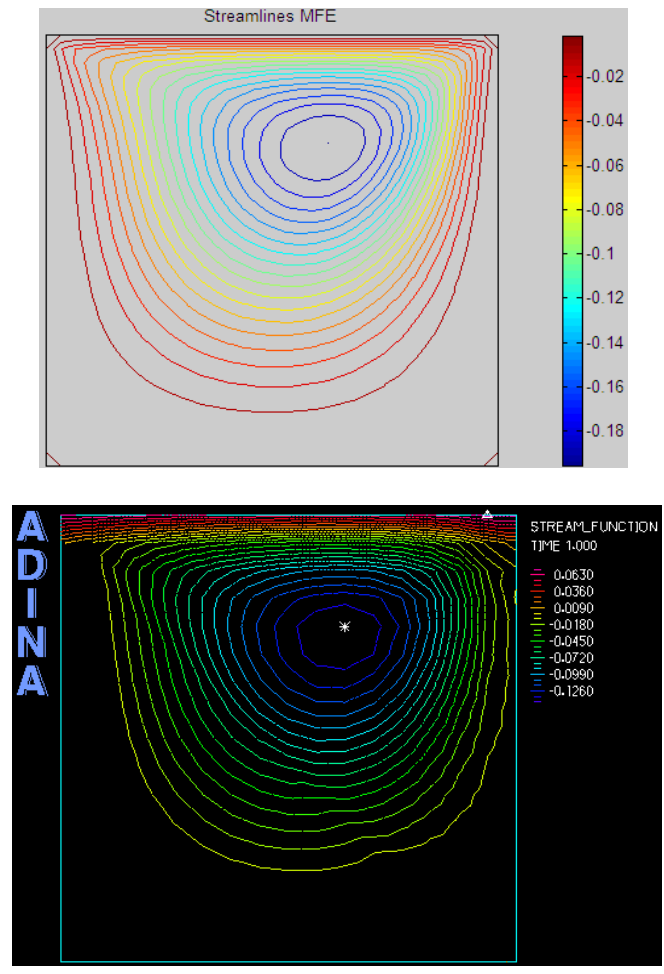

FIGURE 1. Uniform streamline plot with MFE (left), and uniform streamline plot computed with ADINA system (right) using $Q_{1}-P_{0}$ approximation, a $32 \times 32$ square grid and Reynolds number $R e=100$.

TABLE 2. CPU time in seconds for the different approximations with various coarse meshes.

\begin{tabular}{c|c|c|c|c|} 
& $Q_{1}-P_{0}$ & $Q_{1}-Q_{1}$ & $Q_{2}-Q_{1}$ & $Q_{2}-P_{1}$ \\
\hline $8 \times 8$ & 1.2344 & 0.9375 & 1.1406 & 1.2188 \\
$16 \times 16$ & 1.8281 & 1.2813 & 1.7031 & 1.7188 \\
$32 \times 32$ & 4.3281 & 3.1250 & 3.5938 & 4.1094 \\
$64 \times 64$ & 18.7656 & 15.4688 & 23.2969 & 26.1250 \\
\hline
\end{tabular}

Figure 7 shows the iteration counts for GMRES and Bicgstab with leastsquares commutator preconditioning, for linear systems arising from Newton iteration applied to the driven cavity problem with $Q_{1}-P_{0}$ approximation and $R e=100$. 

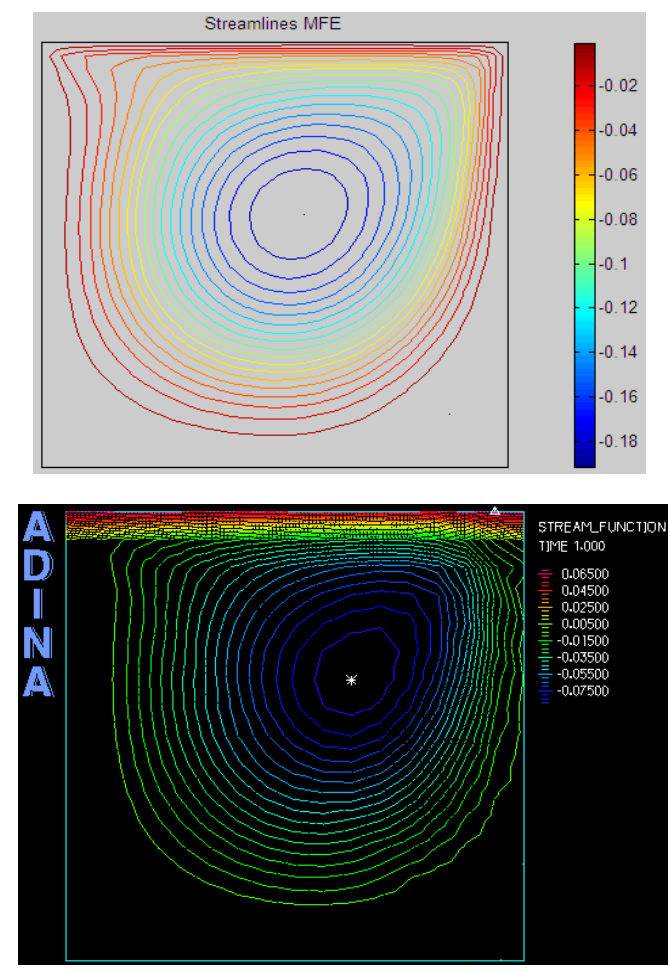

FIGURE 2. Uniform streamline plot with MFE (left), and uniform streamline plot computed with ADINA system (right) using $Q_{1}-P_{0}$ approximation, $32 \times 32$ square grid and Reynolds number $R e=600$.

Some computed errors are given in Table 1 to show the effect of grid refinement in this case. These results, together with the bound (4.35), suggest that the $Q_{1}-P_{0}$ approximation is converging to the exact flow solution at close to optimal rate, that is $O(h)$.

Example 2. $L$-shaped domain $\Omega$, parabolic inflow boundary condition, natural outflow boundary condition.

This example represents flow in a rectangular duct with a sudden expansion; a Poiseuille flow profile is imposed on the inflow boundary $(x=-1 ; 0 \leq y \leq 1)$, and a no-flow (zero velocity) condition is imposed on the walls.

The Neumann condition (5.1) is applied at the outflow boundary $(x=$ $5 ;-1<y<1)$ and automatically sets the mean outflow pressure to zero.

$$
\left\{\begin{array}{l}
\nu \frac{\partial u_{x}}{\partial x}-p=0 \\
\frac{\partial u_{y}}{\partial x}=0
\end{array}\right.
$$



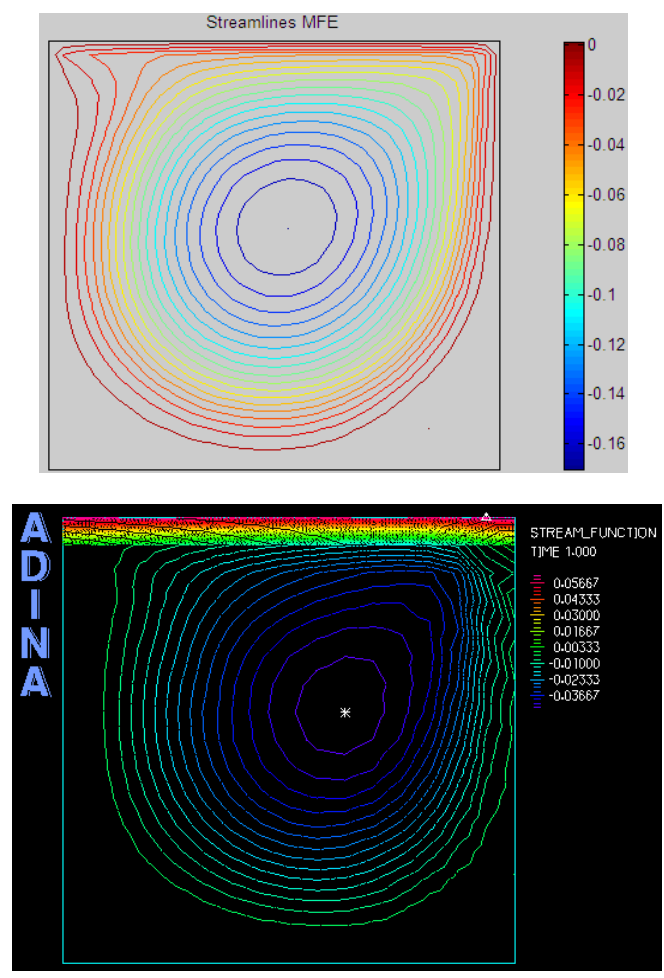

FIGURE 3. Uniform streamline plot with MFE (left), and uniform streamline plot computed with ADINA system (right) using $Q_{1}-P_{0}$ approximation, a $32 \times 32$ square grid and Reynolds number $R e=2000$.

The two solutions are therefore essentially identical. This is very good indication that our solver is implemented correctly.

TABLE 3. Estimated errors for Backward-facing step problem using $Q_{1}-P_{0}$ approximation for the flow with Reynolds number $R e=200$.

\begin{tabular}{c|c|c} 
& $\left\|\nabla \cdot \vec{u}_{h}\right\|_{\Omega}$ & $\eta$ \\
\hline $8 \times 24$ & $3.916 \mathrm{e}-002$ & $8.945 \mathrm{e}+000$ \\
$16 \times 48$ & $1.352 \mathrm{e}-002$ & $5.433 \mathrm{e}+000$ \\
$32 \times 96$ & $4.629 \mathrm{e}-003$ & $3.353 \mathrm{e}-001$ \\
$64 \times 192$ & $1.583 \mathrm{e}-003$ & $2.143 \mathrm{e}-001$ \\
\hline
\end{tabular}

There are two sets of streamlines at equally spaced levels plotted in Figure 8 ; one set is associated with positive streamfunction values and shows the path 

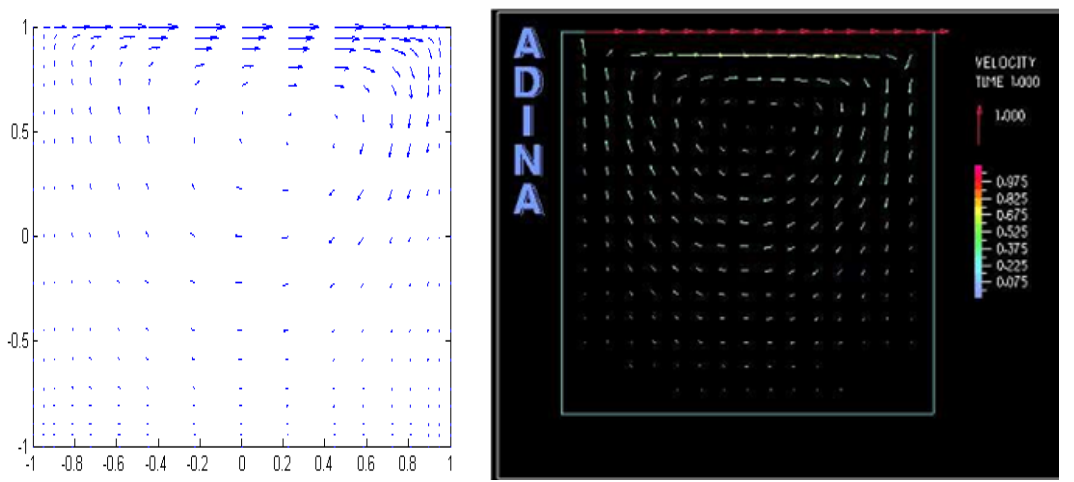

Figure 4. Velocity vectors solution by MFE (left) and Velocity vectors solution computed by ADINA system (right) with a $32 \times 32$ square grid for the flow with Reynolds number $R e=$ 100 .
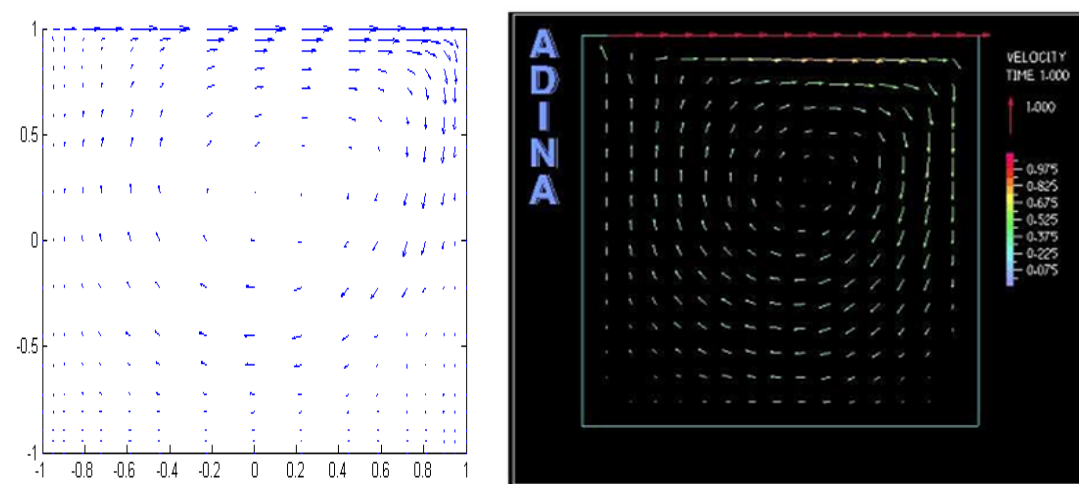

Figure 5 . Velocity vectors solution by MFE (left) and Velocity vectors solution computed by ADINA system (right) with a $32 \times 32$ square grid for the flow with Reynolds number $R e=$ 600 .

of particles introduced at the inflow. These pass over the step and exit at the outflow. The second set of streamlines is associated with negative values of the streamfunction. These streamlines show the path of particles in the recirculation region near the step; they are much closer in value, reflecting the fact that recirculating flow is relatively slow-moving.

If $L$ is taken to be the height of the outflow region, then the flow pattern shown in Figure 8 corresponds to a Reynolds number of 200. If the viscosity parameter were an order of magnitude smaller, then the steady flow would be 

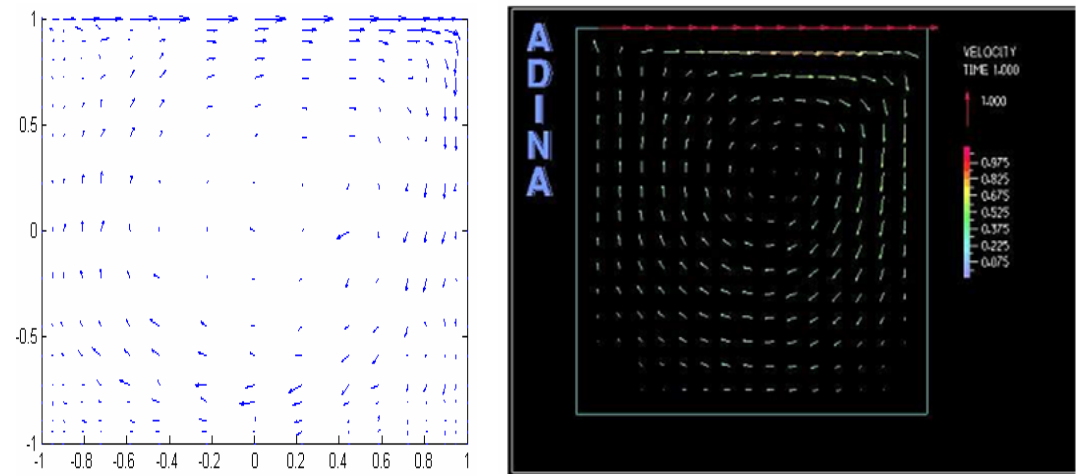

FiguRE 6. Velocity vectors solution by MFE (left) and Velocity vectors solution computed by ADINA system (right) with a $32 \times 32$ square grid for the flow with Reynolds number $R e=$ 2000 .

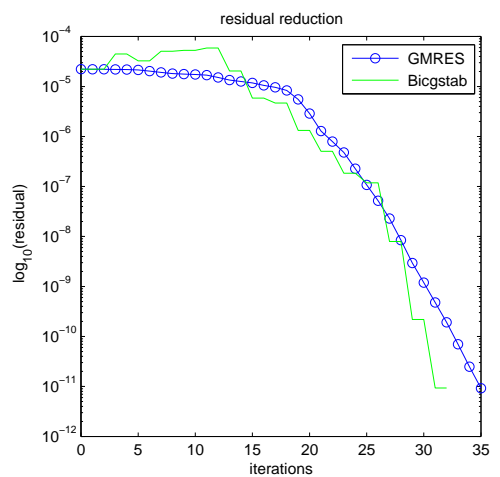

FiguRE 7. Iteration counts for GMRES and Bicgstab with least-squares commutator preconditioning.

unstable. The singularity at the origin is an important feature of the flow even in the convection-dominated case.

\section{Conclusion}

In this work, we were interested in the numerical solution of the partial differential equations by simulating the flow of an incompressible fluid. We applied the mixed finite element method to the resolution of the Navier-Stokes equations. Also, we proposed methods of the estimation of error for the calculated solution. 


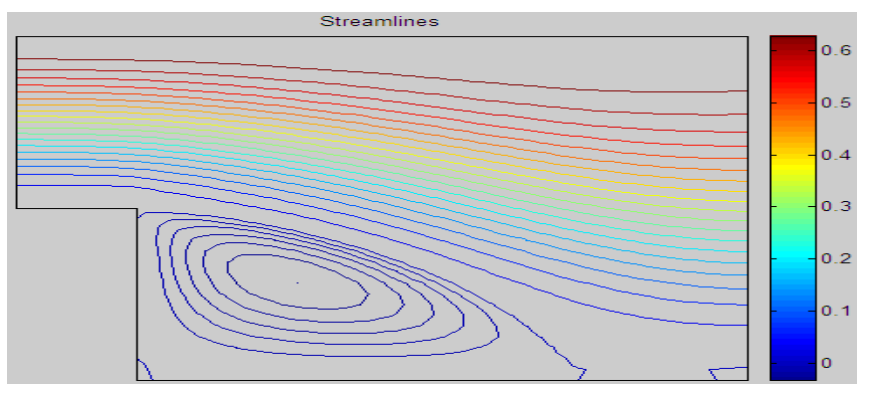

FiguRe 8 . Equally spaced streamline plot associated with a $32 \times 96$ square grid $Q_{1}-P_{0}$ approximation for $\nu=1 / 100$.

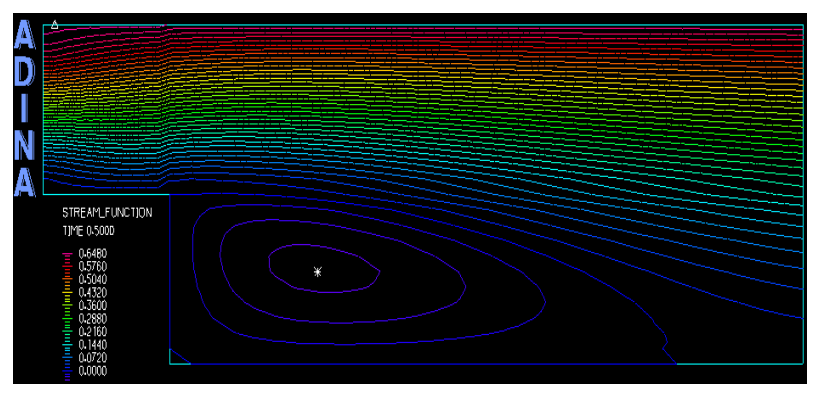

Figure 9. The solution computed with ADINA system. The plots show the Streamlines associated with a $32 \times 96$ square grid, approximation for $\nu=1 / 100$.

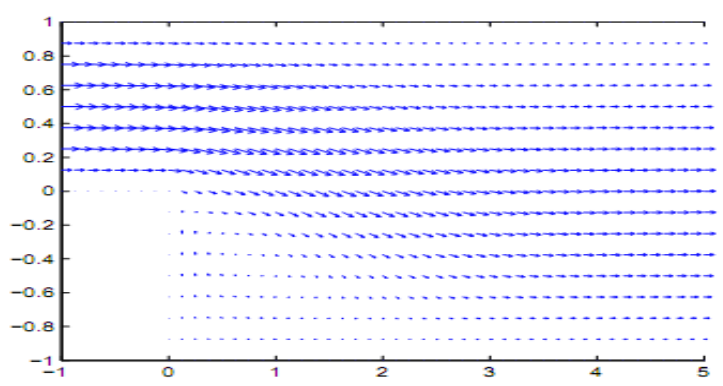

Figure 10. Velocity vectors solution by MFE with a $32 \times 96$ square grid for the flow with $\nu=1 / 100$.

Numerical experiments were carried out and compared with satisfaction with other numerical results, either resulting from the literature, or resulting from calculation with commercial software like Adina system. 


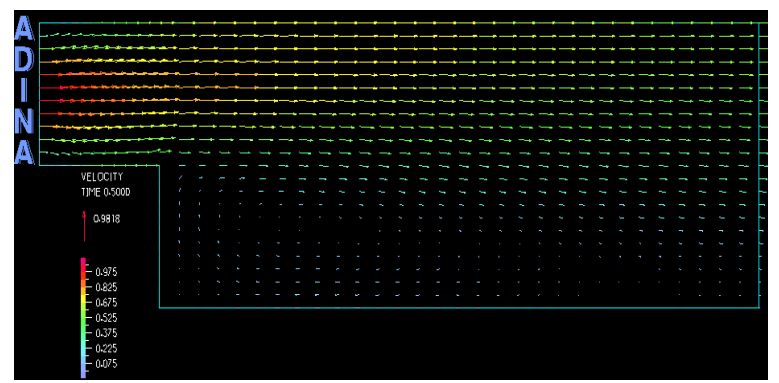

Figure 11. The solution computed with ADINA system. The plots show the velocity vectors solution with a $32 \times 96$ square grid for the flow with $\nu=1 / 100$.

Acknowledgments. The authors would like to express their sincere thanks for the referee for his/her helpful suggestions.

\section{References}

[1] M. Ainsworth and J. Oden, A Posteriori Error Estimation in Finite Element Analysis, Wiley-Interscience [John Wiley \& Sons], New York, 2000.

[2] _ A posteriori error estimators for the Stokes and Oseen equations, SIAM J. Numer. Anal. 34 (1997), no. 1, 228-245.

[3] R. Araya, A. H. Poza, and F. Valentin, On a hierarchical estimator driven by a stabilized method for the reactive incompressible Navier-Stokes equations, Preprint submitted to Elsevier, 2008.

[4] R. E. Bank and A. Weiser, Some a posteriori error estimators for elliptic partial differential equations, Math. Comp. 44 (1985), no. 170, 283-301.

[5] W. Bao and J. W. Barrett, A priori and a posteriori error bounds for a nonconforming linear finite element approximation of a non-Newtonian flow, RAIRO Modél. Math. Anal. Numér. 32 (1998), no. 7, 843-858.

[6] E. Barragy and G. F. Carey, Stream function-vorticity driven cavity solution using $p$ finite elements, Comput. Fluids 26 (1997), 453-468.

[7] M. Benzi and J. Liu, An efficient solver for the incompressible Navier-Stokes equations in rotation form, SIAM J. Sci. Comput. 29 (2007), no. 5, 1959-1981.

[8] F. Brezzi and M. Fortin, Mixed and Hybrid Finite Element Methods, Springer Series in Computational Mathematics, 15. Springer-Verlag, New York, 1991.

[9] C. Carstensen and S. A. Funken, A posteriori error control in low-order finite element discretisations of incompressible stationary flow problems, Math. Comp. 70 (2001), no. 236, 1353-1381.

[10] G. Chavent and J. Jaffré, Mathematical Models and Finite Elements for Reservoir Simulation, Elsevier Science Publishers B. V., Netherlands, 1986

[11] P. G. Ciarlet, Basic error estimates for elliptic problems, Handbook of numerical analysis, Vol. II, 17-351, Handb. Numer. Anal., II, North-Holland, Amsterdam, 1991.

[12] H. C. Elman, Preconditioning for the steady-state Navier-Stokes equations with low viscosity, SIAM J. Sci. Comput. 20 (1999), no. 4, 1299-1316.

[13] H. C. Elman, D. J. Silvester, and A. J. Wathen, Finite Elements and Fast Iterative Solvers: with applications in incompressible fluid dynamics, Oxford University Press, New York, 2005. 
[14] E. Erturk, T. C. Corke, and C. Gokcol, Numerical solutions of 2-D steady incompressible driven cavity flow at high Reynolds numbers, Int. J. Numer. Meth. Fluids 48 (2005), 747-774.

[15] S. Garcia, The lid-driven square cavity flow: from stationary to time periodic and chaotic, Commun. Comput. Phys. 2 (2007), no. 5, 900-932.

[16] A. Gauthier, F. Saleri, and A. Veneziani, A fast preconditioner for the incompressible Navier-Stokes equations, Comput. Vis. Sci. 6 (2004), no. 2-3, 105-112.

[17] U. Ghia, K. Ghia, and C. Shin, High-re solutions for incompressible flow using the Navier-Stokes equations and a multigrid method, J. Comput. Phys. 48 (1982), no. 3, $387-411$.

[18] V. Girault and P. A. Raviart, Finite Element Methods for Navier-Stokes Equations, Springer-Verlag, Berlin, 1986.

[19] P. Gresho, D. Gartling, J. Torczynski, K. Cliffe, K. Winters, T. Garratt, A. Spence, and J. Goodrich, Is the steady viscous incompressible two-dimensional flow over a backwardfacing step at $\mathrm{Re}=800$ stable?, Internat. J. Numer. Methods Fluids 17 (1993), no. 6, 501-541.

[20] P. M. Gresho and R. L. Sani, Incompressible Flow and The finite element Method, John Wiley and Sons, 1998.

[21] J. L. Guermond and L. Quartapelle, On stability and convergence of projection methods based on pressure Poisson equation, Internat. J. Numer. Methods Fluids 26 (1998), no. 9, 1039-1053.

[22] V. John, Residual a posteriori error estimates for two-level finite element methods for the Navier-Stokes equations, Appl. Numer. Math. 37 (2001), no. 4, 503-518.

[23] D. Kay and D. Silvester, A posteriori error estimation for stabilized mixed approximations of the Stokes equations, SIAM J. Sci. Comput. 21 (1999), no. 4, 1321-1336.

[24] M. Li, T. Tang, and B. Fornberg, A compact fourth-order finite difference scheme for the steady incompressible Navier-Stokes equations, Internat. J. Numer. Methods Fluids 20 (1995), no. 10, 1137-1151.

[25] H. Lin and S. N. Atluri, The meshless local Petrov-Galerkin (MLPG) method for solving incompressible Navier-Stokes equations, CMES Comput. Model. Eng. Sci. 2 (2001), no. $2,117-142$.

[26] S. Nicaise, L. Paquet, and Rafilipojaona, A refined mixed finite element method for stationary Navier-Stokes equations with mixed boundary conditions using Lagrange multipliers, Comput. Methods Appl. Math. 7 (2007), no. 1, 83-100.

[27] J. Oden, W. Wu, and M. Ainsworth, An a posteriori error estimate for finite element approximations of the Navier-Stokes equations, Comput. Methods Appl. Mech. Engrg. 111 (1994), no. 1-2, 185-202.

[28] P. A. Raviart and J. M. Thomas, A mixed finite element method for 2nd order elliptic problems, Mathematical aspects of finite element methods (Proc. Conf., Consiglio Naz. delle Ricerche (C.N.R.), Rome, 1975), pp. 292-315. Lecture Notes in Math., Vol. 606, Springer, Berlin, 1977.

[29] M. ur Rehman, C. Vuik, and G. Segal, A comparison of preconditioners for incompressible Navier-Stokes solvers, Internat. J. Numer. Methods Fluids 57 (2008), no. 12, 1731-1751.

[30] J. Roberts and J. M. Thomas, Mixed and Hybrid Methods, Handbook of numerical analysis II, Finite element methods 1, P. Ciarlet and J. Lions, Amsterdam, 1989.

[31] R. Verfürth, A posteriori error estimators for the Stokes equations, Numer. Math. 55 (1989), no. 3, 309-325.

[32] — A Review of a Posteriori Error Estimation and Adaptive Mesh-Refinement Techniques, Wiley-Teubner, Chichester, 1996. 
[33] B. I. Wohlmith and R. H. W. Hoppe, A comparison of a posteriori error estimators for mixed finite element discretizations by Raviart-Thomas elements, Math. Comp. 68 (1999), no. 228, 1347-1378.

[34] D. H. Wu and I. G. Currie, Analysis of a posteriori error indicator in viscous flows, Int. J. Num. Meth. Heat Fluid Flow 12 (2002), no. 3, 306-327.

ABdeslam Elakkad

Laboratoire Génie Mécanique

Faculté des Sciences et Techniques B. P. 2202

Route D'Imouzzer, Fès Maroc

E-mail address: elakkadabdeslam@yahoo.fr

Ahmed Elkhalfi

Laboratoire GÉnie MÉcanique

Faculté des Sciences et Techniques B. P. 2202

Route D'Imouzzer, FÈs Maroc

E-mail address: aelkhalfi@gmail.com

Najib Guessous

DÉPARTEMENT DE MATHÉMATIQUES ET INFORMATIQUE

ECole normale Supérieure de Fès

B. P. 5206, Bensouda, Fès Maroc

E-mail address: ngessous@yahoo.fr 